\title{
Superconductivity and Chiral Symmetry Breaking with Fermion Clusters
}

\author{
Shailesh Chandrasekharan ${ }^{\mathrm{a} *}$

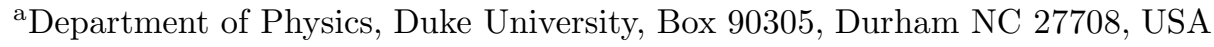

Cluster variables have recently revolutionized numerical work in certain models involving fermionic variables. This novel representation of fermionic partition functions is continuing to find new applications. After describing results from a study of a two dimensional Hubbard type model that confirm a superconducting transition in the Kosterlitz-Thouless universality class, we show how a cluster type algorithm can be devised to study the chiral limit of strongly coupled lattice gauge theories with staggered fermions.

\section{INTRODUCTION}

During the last few years a new class of fermion algorithms have emerged. The essential progress is a result of our ability to rewrite certain fermionic partition functions as a sum over configurations of bond variables with positive definite weights [ㄴ.[2], i.e.,

$$
Z=\sum_{[b]} W[b] \overline{\operatorname{Sign}}[b]
$$

where $W[b]>0$ is the weight of the bond configuration $[b]$ and $\overline{\operatorname{Sign}}[b] \geq 0$ is an entropy factor that takes into account degrees of freedom other than the bond variables. Typically, the Pauli principle is encoded in the topology of clusters formed by lattice sites connected through the bonds. Clusters also carry a variety of interesting physical information. For example, sizes of certain clusters are related to condensates, the squares of the sizes of clusters yield susceptibilities. Further, clusters are useful in building efficient algorithms close to critical points where the correlation lengths diverge since they allow non-local updates with a reasonable acceptance. This property has helped in studying critical phenomena in fermionic models with unmatched precision.

\section{SUPERCONDUCTIVITY}

The recent success of cluster methods in fermionic systems originates from the Hamilto-

\footnotetext{
*This work is supported in part by the Department of
} Energy grant DE-FG02-96DR409845. nian approach. One starts by writing the partition function in terms of configurations of fermion occupation numbers whose Boltzmann weight is not guaranteed to be positive definite. By introducing additional bond variables which help in the integration over the fermion occupation numbers, the partition function of certain models can be rewritten in the form of eq. (11). Configurations with clusters of a specific topology, called merons, then yield $\overline{\operatorname{Sign}}[b]=0$.

Recently, superconductivity in a two dimensional attractive Hubbard type model was studied using the meron cluster approach. The fermion pairing susceptibility $\langle\chi\rangle$ is a useful observable. It is expected to satisfy the finite size scaling formula

$\langle\chi\rangle=\left\{\begin{array}{cc}L^{2-\eta(T)} & T<T_{c} \\ \text { Const. } & T>T_{c}\end{array}\right.$

if the superconducting transition belongs to the Kosterlitz-Thouless (KT) universality class, with $0 \leq \eta(T) \leq 0.25, \eta\left(T_{c}\right)=0.25$ and $\eta(0)=0$. In the specific model studied, $\langle\chi\rangle$ turns out to be a sum over the square of the size of each cluster in the zero meron sector and the product of the size of the merons in the two meron sector. Figure 1 shows a plot of the susceptibility as a function of lattice size for various temperatures. Consistency with KT predictions is clear.

Another observable relevant to the study of superconductivity is the spatial fermion winding number susceptibility $\left\langle W^{2}\right\rangle$. Although this is difficult to evaluate with conventional algorithms, it 


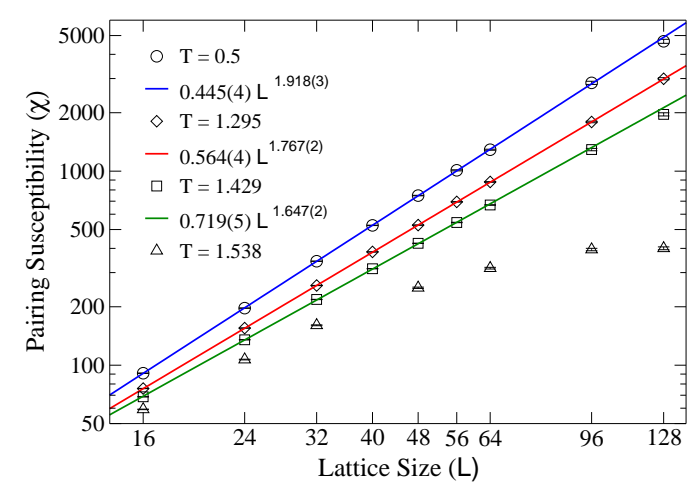

Figure 1. Pairing susceptibility as a function of system size for various temperatures.

is relatively straight forward in the meron cluster approach. Each cluster can be assigned a spatial fermion winding number. The susceptibility then turns out to be the sum over the square of each cluster's spatial winding number in the zero meron sector and the product of the spatial winding number of meron clusters in the two meron sector. In the infinite volume limit below the critical temperature, one can combine known results to obtain $2 \pi \eta(T)\left\langle W^{2}\right\rangle=1$. Results again show consistency with this expectation. Preliminary results from this study was presented in [3] and the final analysis in 4 .

\section{CHIRAL SYMMETRY BREAKING}

Although the recent success has been applied to Hamiltonian models of chiral symmetry breaking [5], cluster methods are applicable to more conventional Lagrangian models as well. For example, consider strongly coupled lattice gauge theory with massless staggered fermions in which the $U(1)$ chiral symmetry is expected to be broken spontaneously in four dimensions [6]. This result was obtained by mapping the massive model into a statistical mechanics of monomer-dimerpolymer (MDP) system with positive definite Boltzmann weights and extrapolating the results to the chiral limit. Unfortunately, as far as we know, it has been difficult to devise algorithms in the chiral limit where the systems become con- strained. Local metropolis updates which can be formulated in the massive case become exponentially inefficient in the chiral limit. Here we argue that cluster representations of the MDP systems yield useful algorithms directly in the chiral limit.

To understand the cluster representation consider for simplicity the strongly coupled $U(1)$ gauge system. The partition function in this case is given by the number of closely-packed-dimer (CPD) configurations on a lattice. A typical CPD configuration in two dimensions is shown in Fig. 2.

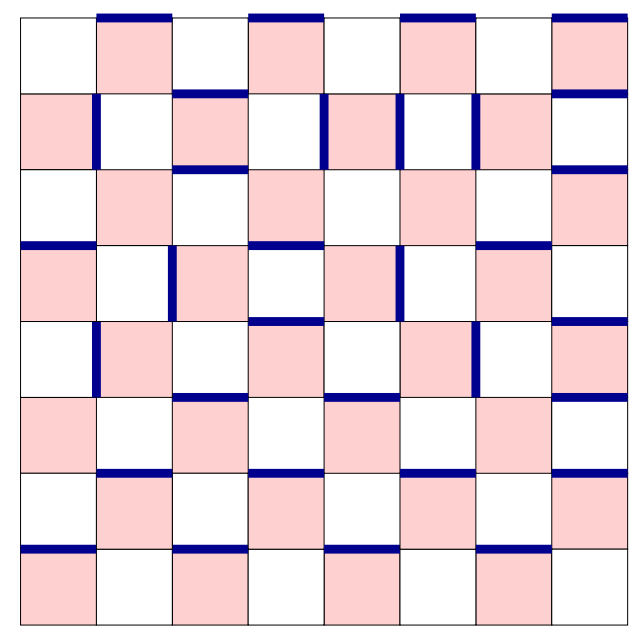

Figure 2. A two dimensional CPD configuration

Such configurations are also of interest in statistical mechanics and play an important role in the solution to the 2-d Ising model [7]. The chiral symmetry of staggered fermions is manifest in this representation by the fact that the chiral condensate vanishes since it is impossible to find a CPD configuration with one defect (one site has no dimer lines attached to it). The chiral susceptibility on the other hand is non-zero and proportional to the ratio of the total number of CPD configurations with two defects (two sites are not connected by dimers) and the partition function.

It is possible to extend CPD configurations 


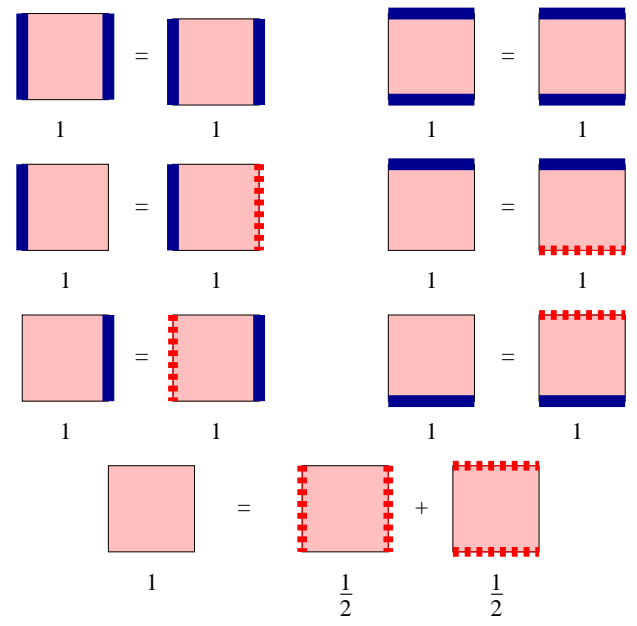

Figure 3. Rules for extending the CPD configurations to include additional bond variables.

to configurations of loops made up of bonds which include the original or "filled" dimers (represented here by "solid" bonds) and "empty" dimers (represented by "dashed" bonds) such that the partition function can be expressed as a sum over weights of new loop configurations. Figure 3 shows the rules of one such extension in two dimensions. Each shaded plaquette of the CPD configuration of Fig. 2 carries one of the seven plaquette configurations given on the left side of equations in Fig. 3. It is easy to check that all constraints are satisfied if each loop is made up of a repeating sequence of filled and empty dimers. The usefulness of the loop variable is that a dimer system can be updated by "flipping" a loop where filled dimers are emptied and vice versa. The acceptance of such a flip is reasonable and leads to a useful algorithm.

The chiral susceptibility gets contributions when a part of the loop is flipped and can be measured easily along with the update. The algorithm was first applied to the two dimensional model. Although a $U(1)$ chiral symmetry cannot spontaneously break in two dimensions, long range correlations can arise as predicted by the Kosterlitz-Thouless universality class as discussed in the previous section. Figure 1 plots the chiral susceptibility with system size. Surprisingly, although the data is not inconsistent with the presence of long range correlations, the susceptibility does not seem to follow the predictions of eq. (2). This puzzle is currently being investigated along with extensions to higher dimensions.

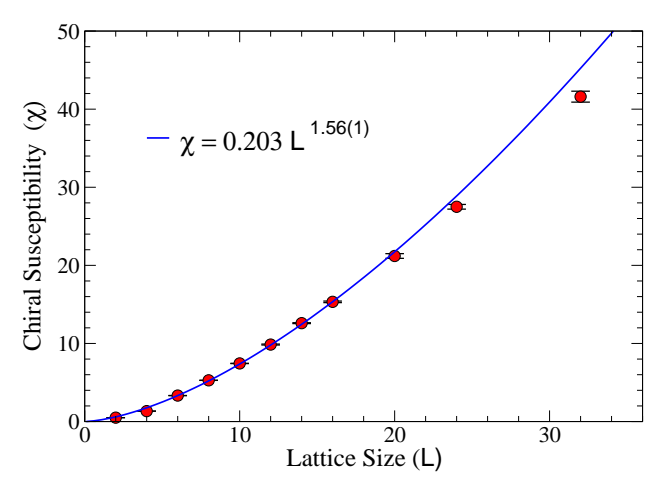

Figure 4. Chiral susceptibility as a function of lattice size in two dimensions.

I would like to thank J. Osborn and U. Wiese for their collaboration.

\section{REFERENCES}

1. S. Chandrasekharan and U.-J. Wiese, Phys. Rev. Lett. 83, (1999) 3116; S. Chandrasekharan, Nucl. Phys. (Proc. Suppl.) 83-84, 774 (2000).

2. See S. Chandrasekharan, hep-lat/0110018 for a recent review.

3. J. C. Osborn, Nucl. Phys. B (Proc. Suppl.) Nucl. Phys. Proc. Suppl. 94, (2001) 865.

4. S. Chandrasekharan and J. C. Osborn, condmat/0109424.

5. S. Chandrasekharan, J. Cox, K. Holland and U.-J. Wiese, Nucl. Phys. B576, 481 (2000);

S. Chandrasekharan and J. C. Osborn, Phys. Lett. B496, 122 (2000).

6. P. Rossi and U. Wolff, Nucl. Phys. B248, (1984) 105.

7. B. M. McCoy and T. T. Wu, Two Dimensional Ising Model", Harvard University Press, Cambridge, Massachusetts, 1973. 